\title{
AN INVERSE EIGENVALUE PROBLEM FOR AN ARBITRARY MULTIPLY CONNECTED BOUNDED REGION IN $\boldsymbol{R}^{2}$
}

\author{
E. M. E. ZAYED \\ Mathematics Department, Faculty of Science \\ Zagazig University \\ Zagazig, Egypt
}

(Received June 26, 1990 and in revised form July 26, 1990)

\begin{abstract}
The basic problem is to determine the geometry of an arbitrary multiply connected bounded region in $R^{2}$ together with the mixed boundary conditions, from the complete knowledge of the eigenvalues $\left\{\lambda_{j}\right\}_{j=1}^{\infty}$ for the Laplace operator, using the asymptotic expansion of the spectral function $\theta(t)=\sum_{j=1}^{\infty} \exp \left(-t \lambda_{j}\right)$ as $t \rightarrow 0$.
\end{abstract}

KEY WORDS AND PHRASES. Inverse problem, Laplace's operator, eigenvalue problem, spectral function.

1980 AMS SUBJECT CLASSIFICATION CODE. 35K, 35P

\section{INTRODUCTION.}

The underlying problem is to deduce the precise shape of a membrane from the complete knowledge of the eigenvalues $\left\{\lambda_{j}\right\}_{j=1}^{\infty}$ for the Laplace operator $\Delta_{2}=\sum_{i=1}^{2}\left(\frac{\partial}{\partial x^{i}}\right)^{2}$ in the $x^{1} x^{2}$-plane.

Let $\Omega \subseteq R^{2}$ be a simply connected bounded domain with a smooth boundary $\partial \Omega$. Consider the Neumann/Dirichlet problem

$$
\begin{gathered}
\left(\Delta_{2}+\lambda\right) u=0 \quad \text { in } \Omega, \\
\frac{\partial u}{\partial n}=0 \text { or } u=0 \text { on } \partial \Omega,
\end{gathered}
$$

where $\frac{\partial}{\partial n}$ denotes differentiation along the inward pointing normal to $\partial \Omega$ and $u \in C^{2}(\Omega) \cap C(\bar{\Omega})$. Denote its eigenvalues, counted according to multiplicity, by

$$
0<\lambda_{1} \leq \lambda_{2} \leq \lambda_{3} \leq \ldots \leq \lambda_{j} \leq \ldots \rightarrow \infty \text { as } j \rightarrow \infty .
$$

The problem of determining the geometry of $\Omega$ has been investigated by Pleijel [1], Kac [2], McKean and Singer [3], Stewartson and Waechter [4], Smith [5], Sleeman and Zayed [6,7], Gottlieb [8], Greiner [9], Zayed [10-13] and the references given there, using the asymptotic expansion of the trace function

$$
\theta(t)=\operatorname{tr}\left[\exp \left(-t \Delta_{2}\right)\right]=\sum_{j=1}^{\infty} \exp \left(-t \lambda_{j}\right) \quad \text { as } \quad t \rightarrow 0 .
$$

It has been shown that, in the case of Neumann boundary conditions (N.b.c.): 


$$
\theta(t)=\frac{|\Omega|}{4 \pi t}+\frac{|\partial \Omega|}{8(\pi t)^{1 / 2}}+a_{0}+\frac{7}{256}\left(\frac{t}{\pi}\right)^{1 / 2} \int_{\partial \Omega} k^{2}(\sigma) d \sigma+0(t) \text { as } t \rightarrow 0,
$$

while, in the case of Dirichlet boundary conditions (D.b.c.):

$$
\theta(t)=\frac{|\Omega|}{4 \pi t}-\frac{|\partial \Omega|}{8(\pi t)^{1 / 2}}+a_{0}+\frac{1}{256}\left(\frac{t}{\pi}\right)^{1 / 2} \int_{\partial \Omega} k^{2}(\sigma) d \sigma+O(t) \text { as } t \rightarrow 0,
$$

In these formulae, $|\Omega|$ is the area of $\Omega,|\partial \Omega|$ is the total length of $\partial \Omega$ and $k(\sigma)$ is the curvature of $\partial \Omega$. The constant term $a_{0}$ has geometric significance, e.g., if $\Omega$ is smooth and convex, then $a_{0}=\frac{1}{6}$ and if $\Omega$ is permitted to have a finite number of smooth convex holes " $H$ ", then $a_{0}=\frac{1}{6}(1-H)$.

The object of this paper is to discuss the following more general inverse problem: Let $\Omega$ be an arbitrary multiply connected bounded region in $R^{2}$ which is surrounded internally by simply connected bounded domains $\Omega_{\imath}$ with smooth boundaries $\partial \Omega_{i}, i=1, \ldots, m-1$ and externally by a simply connected bounded domain $\Omega_{m}$ with a smooth boundary $\partial \Omega_{m}$. Suppose that the eigenvalues (1.3) are given for the eigenvalue equation

$$
\left(\Delta_{2}+\lambda\right) u=0 \text { in } \Omega,
$$

together with one of the following mixed boundary conditions:

$$
\begin{gathered}
\frac{\partial u}{\partial n_{i}}=0 \quad \text { on } \partial \Omega_{i}, \quad i=1, \ldots, k \text { and } u=0 \quad \text { on } \partial \Omega_{i}, \quad i=k+1, \ldots, m, \\
u=0 \quad \text { on } \partial \Omega_{\imath}, \quad i=1, \ldots, k \text { and } \frac{\partial u}{\partial n_{i}}=0 \quad \text { on } \partial \Omega_{i}, \quad i=k+1, \ldots, m,
\end{gathered}
$$

where $\frac{\partial}{\partial n_{i}}$ denote differentiations along the inward pointing normals to the boundaries $\partial \Omega_{i}, i=1, \ldots, m$, respectively.

The basic problem is to determine the geometry of $\Omega$ from the asymptotic expansion of the spectral function (1.4) for small positive $t$.

Note that problems (1.7)-(1.9) have been investigated recently by Zayed [11] in the special case where $\Omega$ is an arbitrary doubly connected bounded region (i.e., $m=2$ ).

\section{STATEMENT OF OUR RESULTS.}

Suppose that the boundaries $\partial \Omega_{i}, i=1, \ldots, m$ are given locally by the equations $x^{n}=y^{n}\left(\sigma_{i}\right), n=1,2$ in which $\sigma_{i}, i=1, \ldots, m$ are the arc-lengths of the counterclockwise oriented boundaries $\partial \Omega_{i}$ and $y^{n}\left(\sigma_{i}\right) \in C^{\infty}\left(\partial \Omega_{i}\right)$. Let $L_{i}$ and $k_{i}\left(\sigma_{i}\right)$ be the lengths and the curvatures of $\partial \Omega_{i}, i=1, \ldots, m$ respectively. Then, the results of our main problem (1.7)-(1.9) can be summarized in the following cases:

CASE 1. (N.b.c. on $\partial \Omega_{i}, i=1, \ldots, k$ and D.b.c. on $\partial \Omega_{\imath}, i=k+1, \ldots, m$ )

$$
\begin{aligned}
\theta(t)= & \frac{|\Omega|}{4 \pi t}+\frac{1}{8(\pi t)^{1 / 2}}\left\{\sum_{i=1}^{k} L_{i}-\sum_{i=k+1}^{m} L_{i}\right\}+\frac{1}{6}(2-m) \\
& +\frac{1}{256}\left(\frac{t}{\pi}\right)^{1 / 2}\left\{7 \sum_{i=1}^{k} \int_{\partial \Omega_{i}} k_{i}^{2}\left(\sigma_{i}\right) d \sigma_{i}+\sum_{i=k+1}^{m} \int_{\partial \Omega_{i}} k_{i}^{2}\left(\sigma_{i}\right) d \sigma_{i}\right\} \\
& +0(t) \quad \text { as } \quad t \rightarrow 0 .
\end{aligned}
$$

CASE 2. (D.b.c. on $\partial \Omega_{i}, i=1, \ldots, k$ and N.b.c. on $\partial \Omega_{i} i=k+1, \ldots, m$ )

In this case the asymptotic expansion of $\theta(t)$ as $t \rightarrow 0$ has the same form (2.1) with the interchanges $\partial \Omega_{i}, i=1, \ldots, k \leftrightarrow \partial \Omega_{i}, i=k+1, \ldots, m$. 
With reference to formulae (1.4), (1.5) and to articles [6], [11], [12] the asymptotic expansion (2.1) may be interpreted as follows:

(i) $\Omega$ is an arbitrary multiply connected bounded region in $R^{2}$ and we have the mixed boundary conditions (1.8) or (1.9) as indicated in the specifications of the two respective cases.

(ii) For the first four terms, $\Omega$ is an arbitrary multiply connected bounded region in $R^{2}$ of area $|\Omega|$. In case 1 , it has $H=(m-1)$ holes, the boundaries $\partial \Omega_{\imath}, i=1, \ldots, k$ are of lengths $\sum_{i=1}^{k} L_{i}$ and of curvatures $k_{\imath}\left(\sigma_{l}\right), i=1, \ldots, k$ together with Neumann boundary conditions, while the boundaries $\partial \Omega_{\imath}, i=k+1, \ldots, m$ are of lengths $\sum_{i=k+1}^{m} L_{i}$ and of curvatures $k_{i}\left(\sigma_{i}\right), i=k+1, \ldots, m$ together with Dirichlet boundary conditions, provided $H$ is an integer.

We close this section with the following remarks:

REMARK 2.1. On setting $k=0$ in formula (2.1) with the usual definition that $\sum_{i=1}^{0}$ is zero, we obtain the results of Dirichlet boundary conditions on $\partial \Omega_{i}, i=1, \ldots, m$.

REMARK 2.2. On setting $k=m$ in formula (2.1) with the usual definition that $\sum_{i=m+1}^{m}$ is zero, we obtain the results of Neumann boundary conditions on $\partial \Omega_{\imath}, i=1, \ldots, m$.

\section{FORMULATION OF THE MATHEMATICAL PROBLEM}

It is easy to show that the spectral function (1.4) associated with problems (1.7)-(1.9) is given by

$$
\theta(t)=\iint_{\Omega} G(\underset{\sim}{x, x ; t}) d x,
$$

where $G\left(x_{1}, x_{2} ; t\right)$ is Green's function for the heat equation

$$
\left(\Delta_{2}-\frac{\partial}{\partial t}\right) u=0
$$

subject to the mixed boundary conditions (1.8) or (1.9) and the initial condition

$$
\lim _{t \rightarrow 0} G\left(\begin{array}{c}
x_{1}, x_{2} ; t \\
-\sim
\end{array}\right)=\delta\left(\begin{array}{c}
x_{1}-x_{2} \\
-
\end{array}\right)
$$

where $\delta\left(\begin{array}{c}x_{1}-x_{2} \\ -\end{array}\right)$ is the Dirac delta function located at the source point $x_{1}=x_{2}$. Let us write

$$
G\left(\underset{\sim}{x_{1}, x_{2} ; t}\right)=G_{0}\left(\underset{\sim}{x_{1}, x_{2} ; t}\right)+\chi\left(\underset{\sim}{x_{1}, x_{2} ; t}\right)
$$

where

$$
G_{0}\left(x_{1}, x_{2} ; t\right)=(4 \pi t)^{-1} \exp \left\{-\frac{\left|\begin{array}{c}
x_{1}-\left.x_{2}\right|^{2} \\
-\sim
\end{array}\right|}{4 t}\right\},
$$

is the "fundamental solution" of the heat equation (3.2), while $\chi\left(\underset{\sim}{\left.x_{1}, x_{2} ; t\right)}\right)$ is the "regular solution" chosen so that $G\left(\underset{\sim}{\left.x_{1}, x_{2} ; t\right)}\right)$ satisfies the mixed boundary conditions (1.8) or (1.9).

On setting $x_{1}=x_{2}=x$ we find that 


$$
\theta(t)=\frac{|\Omega|}{4 \pi t}+K(t)
$$

where

$$
K(t)=\iint_{\Omega} \chi \underset{\sim}{x, x ; t)} \underset{\sim}{x} .
$$

The problem now is to determine the asymptotic expansion of $K(t)$ for small positive $t$. In what follows we shall use Laplace transforms with respect to $t$, and use $s^{2}$ as the Laplace transform parameter; thus we define

$$
\bar{G}\left(\underset{\sim}{\left.x_{1}, x_{2} ; s^{2}\right)}=\int_{0}^{\infty} e^{-s^{2} t} G\left(\underset{\sim}{x_{1}, x_{2} ; t}\right) d t\right.
$$

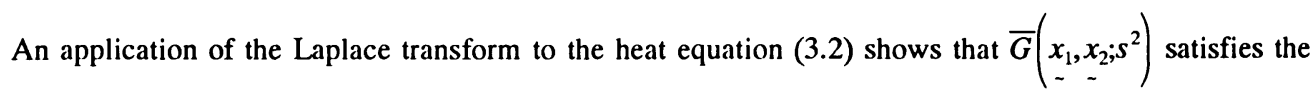
membrane equation

$$
\left(\Delta_{2}-s^{2}\right) \bar{G}\left(\underset{\sim}{x_{1}, x_{2} ; s^{2}}\right)=-\delta\left(\begin{array}{c}
x_{1}-x_{2} \\
\sim
\end{array}\right) \text { in } \Omega,
$$

together with the mixed boundary conditions (1.8) or (1.9).

The asymptotic expansion of $K(t)$ for small positive $t$, may then be deduced directly from the asymptotic expansion of $\bar{K}\left(s^{2}\right)$ for large positive $s$, where

$$
\bar{K}\left(s^{2}\right)=\iint_{\Omega} \bar{\chi}\left(\underset{\sim}{x, x ; s^{2}}\right) d x .
$$

\section{CONSTRUCTION OF GREEN'S FUNCTION.}

It is well known [6] that the membrane equation (3.9) has the fundamental solution

$$
\bar{G}_{0}\left(x_{1}, x_{2} ; s^{2}\right)=\frac{1}{2 \pi} K_{0}\left(s r_{x_{1} x_{2}}\right)
$$

where $r_{x_{1} x_{2}}=\left|\begin{array}{c}x_{1}-x_{2} \\ \sim\end{array}\right|$ is the distance between the points $x_{1}=\left(x_{1}^{1}, x_{1}^{2}\right)$ and $x_{2}=\left(x_{2}^{1}, x_{2}^{2}\right)$ of the region $\Omega$ while $K_{0}$ is the modified Bessel function of the second kind and of zero order. The existence of this solution enables us to construct integral equations for $\bar{G}\left(x_{1}, x_{2} ; s^{2}\right)$ satisfying the mixed boundary conditions (1.8) or (1.9). Therefore, Green's theorem gives:

CASE 1. (N.b.c. on $\partial \Omega_{i}, i=1, \ldots, k$ and D.b.c. on $\partial \Omega_{\imath}, i=k+1, \ldots, m$ )

$$
\begin{aligned}
\bar{G}\left(x_{\sim}, x_{2} ; s^{2}\right)= & \frac{1}{2 \pi} K_{0}\left(s r_{x_{1} x_{2}}\right)+\frac{1}{\pi} \sum_{i=1}^{k} \int_{\partial \Omega_{t}} \bar{G}\left(\underset{\sim}{\left.x_{1}, y ; s^{2}\right)} \frac{\partial}{\partial n_{i y}} K_{0}\left(s r_{y x_{2}}\right) d \underline{-}\right. \\
& +\frac{1}{\pi} \sum_{i=k+1}^{m} \int_{\partial \Omega_{t}} \frac{\partial}{\partial n_{i} y} \bar{G}\left(\underset{\sim}{x_{1}, y ; s^{2}}\right) K_{0}\left(s r_{y x_{2}}\right) d \underset{\sim}{y} .
\end{aligned}
$$

CASE 2. (D.b.c. on $\partial \Omega_{i}, i=1, \ldots, k$ and N.b.c. on $\partial \Omega_{\imath}, i=k+1, \ldots, m$ )

In this case Green's function $\bar{G}\left(x_{1}, x_{2} ; s^{2}\right)$ has the same form (4.2) with the interchanges $\partial \Omega_{\imath}$, $i=1, \ldots, k \leftrightarrow \partial \Omega_{\imath}, i=k+1, \ldots, m$. 
On applying the iteration method (see [11], [12]) to the integral equation (4.2), we obtain Green's function $\bar{G}\left(\underset{\sim}{\left.x_{1}, x_{2} ; s^{2}\right)}\right.$ which has the regular part:

$$
\begin{aligned}
& \bar{\chi}\left(x_{\sim}, x_{2} ; s^{2}\right)=\frac{1}{2 \pi^{2}} \sum_{i=1}^{k} \int_{\partial \Omega_{1}} K_{0}\left(s r_{x_{1} y}\right) \frac{\partial}{\partial n_{i y}} K_{0}\left(s r_{y x_{2}}\right) d \underline{-} \\
& +\frac{1}{2 \pi^{2}} \sum_{i=k+1}^{m} \int_{\partial \Omega_{i}} \frac{\partial}{\partial n_{t y-}} K_{0}\left(s r_{x_{1} y}\right) K_{0}\left(s r_{y x_{2}}\right) d y
\end{aligned}
$$

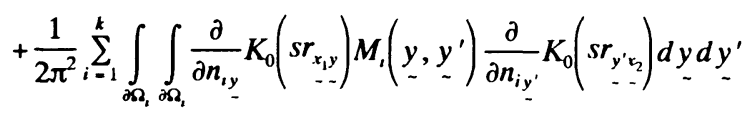

$$
\begin{aligned}
& +\frac{1}{2 \pi^{2}} \sum_{i=k+1}^{m} \int_{\partial \Omega_{1}} \int_{\partial \Omega_{1}} \frac{\partial}{\partial n_{i y}} K_{0}\left(s r_{x_{1} y}\right) M_{i}^{*}\left(\underset{\sim}{y}, \underline{\sim}_{\sim}^{\prime}\right) K_{0}\left(s r_{y^{\prime} x_{2}}\right) d \underline{\sim} \underset{\sim}{y} \underline{y}^{\prime}
\end{aligned}
$$

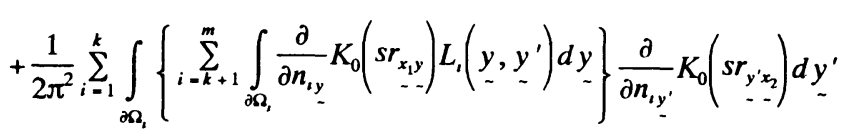

$$
\begin{aligned}
& +\frac{1}{2 \pi^{2}} \sum_{i=k+1}^{m} \int_{\partial \Omega_{1}}\left\{\sum_{i=1}^{k} \int_{\partial \Omega_{1}} K_{0}\left(s r_{x_{1} y^{\prime}}\right) L_{i}^{*}\left(\underset{\sim}{y, y^{\prime}}\right) d \underset{\sim}{y}\right\} K_{0}\left(s r_{y^{\prime} x_{2}}\right) d \underline{y^{\prime}},
\end{aligned}
$$

where

$$
\begin{aligned}
& M_{i}\left(\underset{\sim}{y,} \underset{\sim}{y^{\prime}}\right)=\sum_{v=0}^{\infty} K_{i}^{(v)}\left(\underset{\sim}{y^{\prime}, \underset{\sim}{y}}\right), \\
& M_{i}^{*}\left(\underset{\sim}{y}, \underline{\sim}^{\prime}\right)=\sum_{v=0}^{\infty} K_{i}^{(v)}\left(\underline{\sim}^{y^{\prime}}, \underset{\sim}{y}\right), \\
& L_{\imath}\left(\underset{\sim}{y}, \underline{\sim}^{\prime}\right)=\sum_{v=0}^{\infty} \underline{K}_{i}^{(v)}\left(\underline{\sim}^{\prime}, \underline{y}\right), \\
& L_{i}^{*}\left(\underset{\sim}{y}, \underline{\sim}^{\prime}\right)=\sum_{v=0}^{\infty} \underline{K}_{i}^{(v)}\left(\underset{\sim}{y^{\prime}, y}\right) \text {, } \\
& K_{i}\left(\underset{\sim}{\left.y^{\prime}, \underset{\sim}{y}\right)}\right)=\frac{1}{\pi} \frac{\partial}{\partial n_{i y}} K_{0}\left(s r_{y y^{\prime}}\right), \\
& K_{i}\left(\underset{\sim}{\left.y^{\prime},{ }_{\sim}^{y}\right)}\right)=\frac{1}{\pi} \frac{\partial}{\partial n_{i y^{\prime}}} K_{0}\left(s r_{y y^{\prime}}\right), \\
& \underline{K}_{i}\left(\underline{\sim}^{y^{\prime}, y}\right)=\frac{1}{\pi} K_{0}\left(s r_{y y^{\prime}}\right),
\end{aligned}
$$

and

$$
\underline{K}_{i}\left(\underset{\sim}{\left.y^{\prime}, \underset{\sim}{y}\right)}\right)=\frac{1}{\pi} \frac{\partial^{2}}{\partial n_{i \underline{y}} \partial n_{i y^{\prime}}} K_{0}\left(s r_{y \underline{y}^{\prime}}\right) .
$$

In the same way, we can show that in case 2 Green's function $\bar{G}\left(x_{1}, x_{2} ; s^{2}\right)$ has a regular part of the same form (4.3) with the interchanges $\partial \Omega_{\imath}, i=1, \ldots, k \leftrightarrow \partial \Omega_{i}, i=k+1, \ldots, m$. 
On the basis of (4.3) the function $\bar{\chi}\left(x_{\sim}, x_{2} ; s^{2}\right)$ will be estimated for large values of $s$. The case when $x_{1}$ and $x_{2}$ lie in the neighborhoods of $\partial \Omega_{l}, i=1, \ldots, m$ is particularly interesting. For this case, we need to use the following coordinates.

5. COORDINATES IN THE NEIGHBORHOODS OF $\partial \Omega_{\imath}, i=1, \ldots, m$.

Let $n_{i}, i=1, \ldots, m$ be the minimum distances from a point $x=\left(\underline{\sim}^{1}, x^{2}\right)$ of the region $\Omega$ to the boundaries $\partial \Omega_{i}, i=1, \ldots, m$ respectively. Let $n_{i}\left(\sigma_{t}\right), i=1, \ldots, m$ denote the inward drawn unit normals to $\partial \Omega_{\imath}, i=1, \ldots, m$ respectively. We note that the coordinates in the neighborhood of $\partial \Omega_{\imath}, i=k+1, \ldots, m$ and its diagrams (see [11]) are in the same form as in section 5.1 of [11] with the interchanges $\sigma_{2} \leftrightarrow \sigma_{1}$, $n_{2} \leftrightarrow n_{t}, h_{2} \leftrightarrow h_{t}, I_{2} \leftrightarrow I_{t}, \mathcal{D}\left(I_{2}\right) \leftrightarrow \mathcal{D}\left(I_{i}\right)$ and $\delta_{2} \leftrightarrow \delta_{t}, i=k+1, \ldots, m$. Thus, we have the same formulae (5.1.1)-(5.1.5) of section 5.1 in [11] with the interchanges $n_{2} \leftrightarrow n_{1}, n_{2}\left(\sigma_{2}\right) \leftrightarrow n_{\sim}\left(\sigma_{i}\right), t_{2}\left(\sigma_{2}\right) \leftrightarrow t_{\sim}\left(\sigma_{1}\right)$, $k_{2}\left(\sigma_{2}\right) \leftrightarrow k_{\imath}\left(\sigma_{\imath}\right), \quad i=k+1, \ldots, m$.

Similarly, the coordinates in the neighborhood of $\partial \Omega_{t}, i=1, \ldots, k$ and its diagrams (see [11]) are similar to those obtained in section 5.2 of [11] with the interchanges $\sigma_{1} \leftrightarrow \sigma_{i}, n_{1} \leftrightarrow n_{i}, h_{1} \leftrightarrow h_{i}, I_{1} \leftrightarrow I_{i}$, $\mathcal{D}\left(I_{1}\right) \leftrightarrow \mathcal{D}\left(I_{i}\right)$ and $\delta_{1} \leftrightarrow \delta_{i}, i=1, \ldots, k$. Thus, we have the same formulae (5.2.1)-(5.2.5) of section 5.2 in [11] with the interchanges $n_{1} \leftrightarrow n_{1}, n_{1}\left(\sigma_{1}\right) \leftrightarrow n_{i}\left(\sigma_{1}\right), t_{1}\left(\sigma_{1}\right) \leftrightarrow t_{1}\left(\sigma_{1}\right)$ and $k_{1}\left(\sigma_{1}\right) \leftrightarrow k_{1}\left(\sigma_{i}\right), i=1, \ldots, k$.

\section{SOME LOCAL EXPANSIONS.}

It now follows that the local expansions of the functions

$$
K_{0}\left(s r_{x y-}\right), \quad \frac{\partial}{\partial n_{t y}} K_{0}\left(s r_{x y}\right), \quad i=1, \ldots, m
$$

when the distance between $x$ and $y$ is small, are very similar to those obtained in section 6 of [11]. Consequently, for $i=1, \ldots, k, k+1, \ldots, m$, the local behavior of the following kernels:

$$
\begin{aligned}
& K,\left(\underset{\sim}{y^{\prime}, y}\right), \quad \underline{K}_{-}\left({\left.\underset{\sim}{y^{\prime}}, \underset{\sim}{y}\right)}^{\prime},\right. \\
& K_{i}\left(\underset{\sim}{y^{\prime}}, \underline{-}\right), \underline{K},\left(\underset{\sim}{y^{\prime}, y}\right),
\end{aligned}
$$

when the distance between $y$ and $y^{\prime}$ is small, follows directly from the knowledge of the local expansions of (6.1).

DEFINITION 1. Let $\xi_{1}$ and $\xi_{2}$ be points in the upper half-plane $\xi^{2}>0$, then we define

$$
\hat{\rho}_{12}=\sqrt{\left(\xi_{1}^{1}-\xi_{2}^{l}\right)^{2}+\left(\xi_{1}^{2}+\xi_{2}^{2}\right)^{2}} .
$$

An $e^{\lambda}\left(\underset{\sim}{\xi_{1}}, \xi_{2} ; s\right)$-function is defined for points $\underline{\sim}_{1}$ and $\xi_{2}$ belong to sufficiently small domains $\mathcal{D}\left(I_{1}\right)$ except when $\xi_{1}=\xi_{2} \in I_{i}, i=1, \ldots, m$ and $\lambda$ is called the degree of this function. For every positive integer $\Lambda$ it has the local expansion (see [11]): 


$$
e^{\lambda}\left(\underline{\xi}_{1}, \xi_{2} ; s\right)=\sum^{*} f\left(\xi_{1}^{1}\right)\left(\xi_{1}^{2}\right)^{P_{1}}\left(\xi_{2}^{2}\right)^{P_{2}}\left(\frac{\partial}{\partial \xi_{1}^{1}}\right)^{l}\left(\frac{\partial}{\partial \xi_{1}^{2}}\right)^{m} K_{0}\left(s \hat{\rho}_{12}\right)+R^{\wedge}\left(\underline{\xi}_{1}, \xi_{2}, s\right),
$$

where $\Sigma^{*}$ denotes a sum of a finite number of terms in which $f\left(\xi_{1}^{1}\right)$ is an infinitely differentiable function. In this expansion, $P_{1}, P_{2}, l, m$ are integers, where $P_{1} \geq 0, P_{2} \geq 0, l \geq 0, \lambda=\min \left(P_{1}+P_{2}-q\right), q=l+m$ and

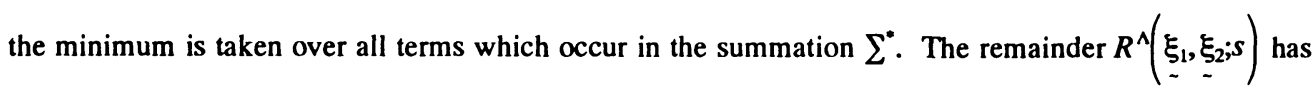
continuous derivatives of order $d \leq \Lambda$ satisfying

$$
D^{d} R^{\Lambda}\left(\underset{\sim}{\xi_{1}}, \underline{\sim}_{2} ; s\right)=0\left(s^{-\Lambda} e^{-A s \hat{1}_{12}}\right) \text { as } s \rightarrow \infty
$$

where $A$ is a positive constant.

Thus, using methods similar to those obtained in section 7 of [11], we can show that the functions (6.1) are $e^{\lambda}$-functions with degrees $\lambda=0,-1$ respectively. Consequently, the functions (6.2) are $e^{\lambda}$-functions with degrees $\lambda=0,-1$, while the functions (6.3) are $e^{\lambda}$-functions with degrees $\lambda=0,1$ respectively.

DEFINITION 2. If $x_{1}$ and $x_{2}$ are points in large domains $\Omega+\partial \Omega_{i}, i=1, \ldots, k, k+1, \ldots, m$, then we define

$$
\hat{r}_{12}=\min _{\underline{y}}\left(r_{x_{1} y}+r_{x_{2} y}\right) \text { if } y \in \partial \Omega_{i}, \quad i=1, \ldots, k,
$$

and

$$
\hat{R}_{12}=\min _{\underline{y}}\left(r_{x_{1} y}+r_{x_{2} y}\right) \quad \text { if } \quad \underline{\sim} \in \partial \Omega_{i}, \quad i=k+1, \ldots, m .
$$

An $E^{\lambda}\left(x_{1}, x_{2} ; s\right)$-function is defined and infinitely differentiable with respect to $x_{1}$ and $x_{2}$ when these points belong to large domains $\Omega+\partial \Omega_{i}$ except when $x_{1}=x_{2} \in \partial \Omega_{i}, i=1, \ldots, m$. Thus, the $E^{\lambda}$-function has a similar local expansion of the $e^{\lambda}$-function (see [6], [11]).

By the help of section 8 in [11], it is easily seen that formula (4.3) is an $E^{0}\left(x_{1}, x_{2} ; s\right)$-function and consequently

$$
\begin{aligned}
\bar{G}\left(x_{1}, x_{2} ; s^{2}\right)= & \sum_{i=1}^{k} O\left\{\left[1+\left|\log s \hat{r}_{12}\right|\right] e^{-\lambda_{i} s \hat{s}_{12}}\right\}, \\
& +\sum_{i=k+1}^{m} O\left\{\left[1+\left|\log s \hat{R}_{12}\right|\right] e^{-A_{i} s \hat{R}_{12}}\right\},
\end{aligned}
$$

which is valid for $s \rightarrow \infty$, where $A_{i}, i=1, \ldots, m$ are positive constants.

Formula (6.7) shows $\bar{G}\left(\underset{\sim}{x_{1}, x_{2} ; s^{2}}\right)$ is exponentially small for $s \rightarrow \infty$.

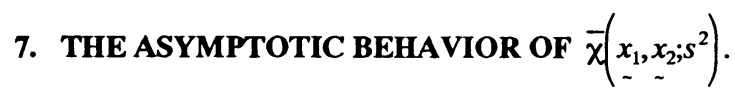

With reference to sections 7 and 9 in [11], if the $e^{\lambda}$-expansions of the functions (6.1)-(6.3) are introduced into (4.3) and if we use formulae similar to (7.4) and (7.10) of section 7 in [11], we obtain the following local behavior of $\bar{\chi}\left(x_{\sim}, x_{2} ; s^{2}\right)$ as $s \rightarrow \infty$ which is valid when $\hat{r}_{12}$ and $\hat{R}_{12}$ are small:

$$
\bar{\chi}\left(x_{\sim}, x_{2} ; s^{2}\right)=\sum_{i=1}^{m} \bar{\chi}_{1}\left(x_{\sim}, x_{2} ; s^{2}\right)
$$


where, if $x_{1}$ and $x_{2}$ belong to sufficiently small domains $\mathcal{D}\left(I_{i}\right), i=1, \ldots, k, k+1, \ldots, m$, then

$$
\bar{\chi}_{i}\left(x_{1}, x_{2} ; s^{2}\right)=-\frac{1}{2 \pi} K_{0}\left(s \hat{\rho}_{12}\right)+O\left\{s^{-1} \exp \left(-A_{t} s \hat{\rho}_{12}\right)\right\}
$$

When $\hat{r}_{12} \geq \delta_{i}>0, i=1, \ldots, k$ and $\hat{R}_{12} \geq \delta_{\imath}>0, i=k+1, \ldots, m$ the function $\bar{\chi}\left(x_{1}, x_{2} ; s^{2}\right)$ is of order $O\{\exp (-c s)\}$ as $s \rightarrow \infty, c>0$. Thus, since $\lim _{\hat{\hat{P}}_{12} \rightarrow 0} \frac{\hat{\hat{f}}_{12}}{\hat{\mathrm{P}}_{12}}=\lim _{\hat{R}_{12} \rightarrow 0} \frac{\hat{R}_{12}}{\hat{\hat{P}}_{12}}=1$, then if $x_{1}$ and $x_{2}$ belong to large domains $\Omega+\partial \Omega_{i}, i=1, \ldots, k$, we deduce for $s \rightarrow \infty$ that

$$
\bar{\chi}_{i}\left(x_{\sim}^{x_{1}, x_{2} ; s^{2}}\right)=-\frac{1}{2 \pi} K_{0}\left(s \hat{r}_{12}\right)+O\left\{s^{-1} \exp \left(-A_{i} s \hat{r}_{12}\right)\right\},
$$

while, if $x_{1}$ and $x_{2}$ belong to large domains $\Omega+\partial \Omega_{i}, i=k+1, \ldots, m$, we deduce for $s \rightarrow \infty$ that

$$
\bar{\chi}_{\imath}\left(x_{1}, x_{2} ; s^{2}\right)=-\frac{1}{2 \pi} K_{0}\left(s \hat{R}_{12}\right)+O\left\{s^{-1} \exp \left(-A_{t} s \hat{R}_{12}\right)\right\} .
$$

\section{CONSTRUCTION OF OUR RESULTS.}

Since for $\xi^{2} \geq h_{i}>0, i=1, \ldots, k, k+1, \ldots, m$, the functions $\bar{\chi}_{i}\left(\underset{\sim}{x, x ; s^{2}}\right)$ are of order $O\left\{\exp \left(-2 s A_{i} h_{i}\right)\right\}$, the integral of the function $\bar{\chi}\left(\underset{\sim}{x, x} ; s^{2}\right)$ over the region $\Omega$ can be approximated in the following way (see (3.10)):

$$
\begin{aligned}
& \bar{K}\left(s^{2}\right)=\sum_{i=k+1}^{m} \int_{\xi^{2}=0}^{h_{i}} \int_{\xi^{1}=0}^{L_{i}} \bar{\chi}_{i}\left(\underset{\sim}{x, x ; s^{2}}\right)\left\{1-k_{t}\left(\xi^{1}\right) \xi^{2}\right\} d \xi^{1} d \xi^{2} \\
& -\sum_{i=1}^{k} \int_{\xi^{2}=0}^{h_{i}} \int_{\xi^{1}=0}^{L_{1}} \bar{\chi}_{i}\left(\underset{-}{x, x ; s^{2}}\right)\left\{1+k_{1}\left(\xi^{1}\right) \xi^{2}\right\} d \xi^{1} d \xi^{2} \\
& +\sum_{i=1}^{m} O\left\{\exp \left(-2 s A_{i} h_{i}\right)\right\} \quad \text { as } \quad s \rightarrow \infty .
\end{aligned}
$$

If the $e^{\lambda}$-expansions of $\bar{\chi}_{i}\left(\underset{\sim}{x, x} ; s^{2}\right), i=1, \ldots, k, k+1, \ldots, m$, are introduced into (8.1), one obtains an asymptotic series of the form:

$$
\bar{K}\left(s^{2}\right)=\sum_{n=1}^{j} a_{n} s^{-n}+O\left(s^{-j-1}\right) \text { as } s \rightarrow \infty,
$$

where the coefficients $a_{n}$ are calculated from the $e^{\lambda}$-expansions by the help of formula (10.3) of section 10 in [11].

Now, the first three coefficients $a_{1}, a_{2}, a_{3}$ take the forms: 


$$
\begin{aligned}
& a_{1}=\frac{1}{8}\left(\sum_{i=1}^{k} L_{t}-\sum_{i=k+1}^{m} L_{t}\right), \\
& a_{2}=\frac{1}{6}(2-m), \\
& a_{3}=\frac{1}{512}\left\{7 \sum_{i=1}^{k} \int_{\partial \Omega_{i}} k_{i}^{2}\left(\sigma_{t}\right) d \sigma_{i}+\sum_{i=k+1}^{m} \int_{\partial \Omega_{i}} k_{i}^{2}\left(\sigma_{i}\right) d \sigma_{i}\right\} .
\end{aligned}
$$

On inserting (8.3) int (8.2) and inverting Laplace transforms and using (3.6) we arrive at our result (2.1).

\section{REFERENCES}

[1] PLEIJEL, A. A study of certain Green's functions with applications in the theory of vibrating membranes, Ark. Mat. $\underline{2}$ (1954), 553-569.

[2] KAC, M. Can one hear the shape of a drum? Amer. Math. Month, 73, No. 4, Part II (1966), 1-23.

[3] McKEAN, H. P. and SINGER, I. M. Curvature and the eigenvalues of the Laplacian, J. Diff. Geometry, 1 (1967), 43-69.

[4] STEWARTSON, K. and WAECHTER, R. T. On hearing the shape of a drum: further results, Proc. Camb. Phil. Soc. 69 (1971), 353-363.

[5] SMITH, L. The asymptotics of the heat equation for a boundary value problem, Invent. Math $\underline{63}$ (1981), 467-493.

[6] SLEEMAN, B. D. and ZAYED, E. M. E. An inverse eigenvalue problem for a general convex domain, J. Math. Anal. Appl. 94 (1983), 78-95.

[7] SLEEMAN, B. D. and ZAYED, E. M. E. Trace formulae for the eigenvalues fo the LaPlacian, J. Applied. Math. Phys., 35 (1984), 106-115.

[8] GOTTLIEB, H. P. W. Eigenvalues of the Laplacian for rectilinear regions, J. Austral. Math. Soc. Ser. B 29 (1988), 270-281.

[9] GREINER, P. An asymptotic expansion for the heat equation, Arch. Rational. Mech. Anal. 41 (1971), 163-218.

[10] ZAYED, E. M. E. Eigenvalues of the Laplacian for the third boundary value problem, J.Austral. Math. Soc. Ser. B. 29 (1987), 79-87.

[11] ZAYED, E. M. E. Heat equation for an arbitrary doubly-connected region in $R^{2}$ with mixed conditions, J. Applied Math. Phys. 40 (1989), 339-355.

[12] ZAYED, E. M. E. Hearing the shape of a general convex domain, J. Math. Anal. Appl. 142 (1989), 170-187.

[13] ZAYED, E. M. E. On hearing the shape of an arbitrary doubly-connected region in $R^{2}$, J.Austral. Math. Soc, Ser. B31, No. 4 (1990), 472-483.

*Present address: Mathematics Department

Faculty of Science

University of Emirates

P.O. Box 15551

Al-Ain, United Arab Emirates 


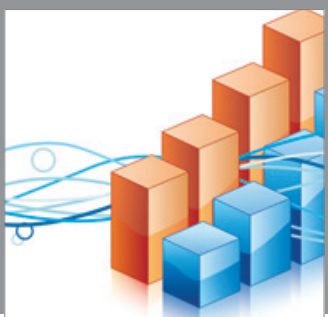

Advances in

Operations Research

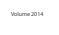

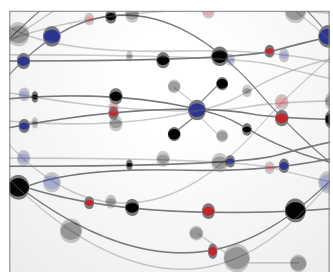

\section{The Scientific} World Journal
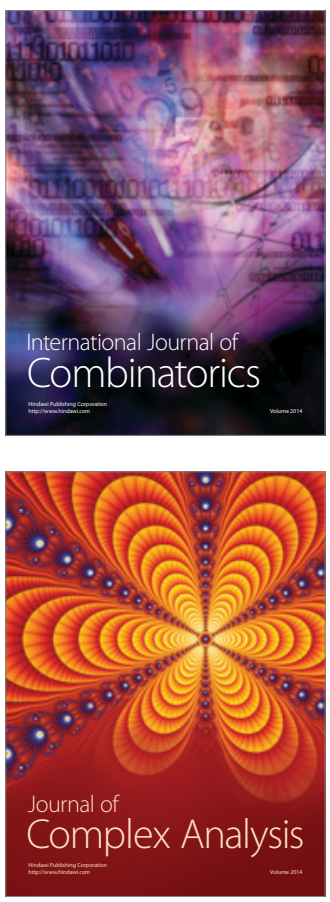

International Journal of

Mathematics and

Mathematical

Sciences
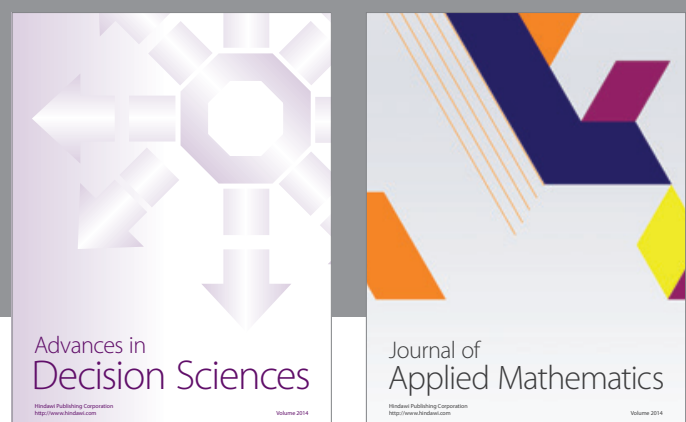

Journal of

Applied Mathematics
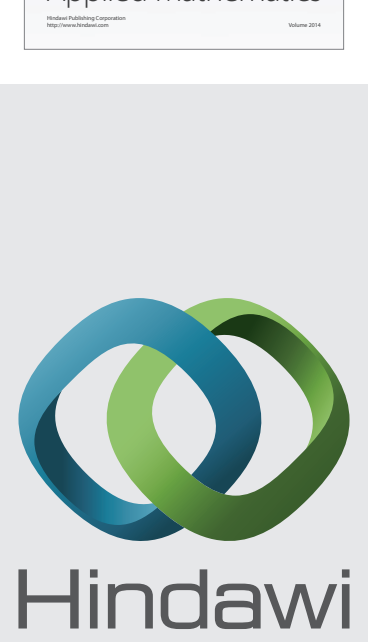

Submit your manuscripts at http://www.hindawi.com
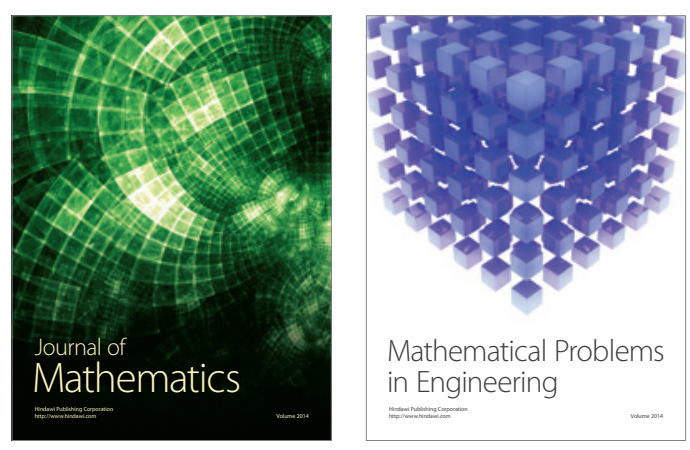

Mathematical Problems in Engineering
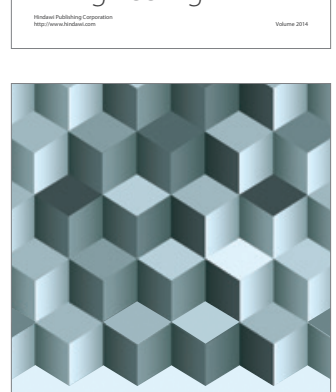

Journal of

Function Spaces
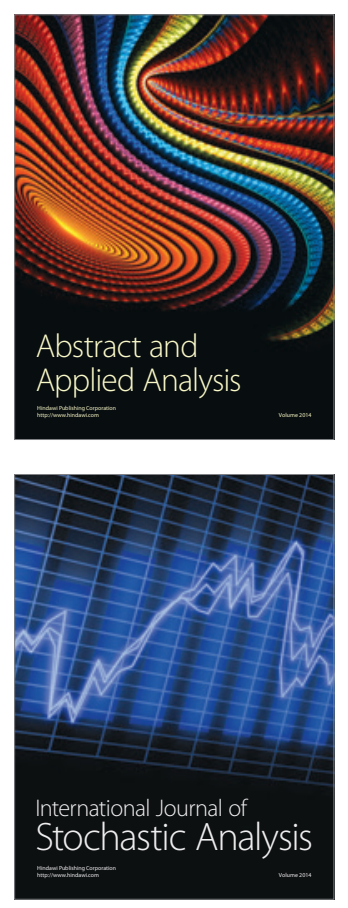

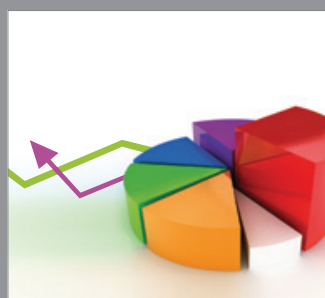

ournal of

Probability and Statistics

Promensencen
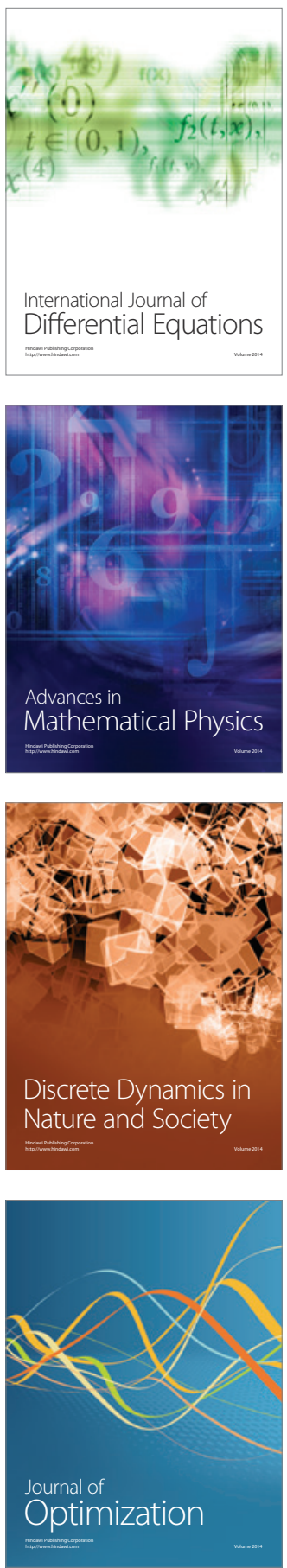